\title{
AVANÇOS NA PRODUÇÃO DE PÃES SEM GLÚTEN: ASPECTOS TECNOLÓGICOS E NUTRICIONAIS
}

\begin{abstract}
Esta revisão de literatura teve como objetivo levantar informações sobre a produção de pães sem glúten, com ênfase nos aspectos tecnológicos e nutricionais. As abordagens tecnológicas incluiram o uso de amidos, hidrocoloides, proteínas, enzimas, emulsificantes e suas combinações com o objetivo de melhorar a estrutura, a textura, a aceitabilidade e a vida-de-prateleira de pães sem glúten. Foram apresentadas pesquisas sobre a adição de fibras alimentares, proteínas, minerais e farinhas de grãos integrais para melhorar o valor nutritivo desses produtos. Verificou-se a necessidade de mais pesquisa e desenvolvimento nessa área, facilitando o acesso dos pacientes celíacos a pães isentos de glúten com qualidade tecnológica e nutricional adequada, contribuindo para maior adesão à dieta específica e melhorando a qualidade de vida desses indivíduos.
\end{abstract}

* Doutora em Ciências, Professora Adjunta, Departamento de Ciências da Saúde, Campus Baixada Santista, Universidade Federal de São Paulo (UNIFESP), Santos, SP (e-mail: vancapri@usp.br).

** Doutor em Ciência dos Alimentos, Professor titular, Departamento de Nutrição, Faculdade de Saúde Pública, USP, São Paulo, SP (e-mail: jagareas@usp.br). 


\section{INTRODUÇÃo}

A doença celíaca, enfermidade crônica, é causada pela intolerância permanente à ingestão das proteínas de estocagem do trigo, da cevada e do centeio, denominadas genericamente como glúten (REWERS, 2005). Estima-se que as taxas de prevalência dessa doença em populações ocidentais variem de 1:50 a 1:300 (HOFFENBERG et al., 2003).

A doença não tem cura e apresenta como único tratamento a remoção do glúten da dieta, tanto para os indivíduos sintomáticos como para os assintomáticos. Essa restrição alimentar deve ser mantida por toda a vida, prevenindo complicações em curto e longo prazos, como os prejuízos nutricionais, de crescimento e desenvolvimento psicomotor, desenvolvimento sexual e problemas de fertilidade, alterações dentárias, osteopenia, osteoporose e anemias, atrofia do baço e cânceres do tubo digestivo (SÁNCHEZ et al., 1992; FERGUSON e KINGSTONE, 1996; REWERS, 2005). Os cânceres são responsáveis pelo aumento da mortalidade desses pacientes.

A forte adesão à dieta parece ser a única possibilidade de prevenir certos tipos raros e agressivos de cânceres (CATASSI, BEARZI e HOLMES, 2005). Entretanto, a remoção do glúten da dieta é problemática. Muitos dos alimentos normalmente consumidos e de custo relativamente baixo, tais como pão, macarrão e biscoitos apresentam glúten em sua formulação. São frequentes as dificuldades para dar sequência ao tratamento, devido à escassez e ao elevado custo dos produtos isentos de glúten, além da aquisição e preparo de produtos que não fazem parte do hábito familiar (EGASHIARA et al., 1996; LEE e NEWMAN 2003; RASHID et al., 2003; D'AMICO et al., 2005).

Andreoli (2003) observou transgressão à dieta em 46\% das crianças e em 35,5\% dos adolescentes atendidos num ambulatório de gastroenterologia no Brasil. Resultados parecidos foram observados em crianças americanas celíacas, sendo que $51 \%$ delas revelaram transgredir a dieta intencionalmente (D'AMICO et al., 2005).

Produtos específicos são indispensáveis para a melhoria da qualidade de vida dos pacientes celíacos. Resultados de inquérito realizado pela Associação de Celíacos do Brasil revelaram que 47\% dos pacientes gostariam de encontrar pão sem glúten com maior facilidade (ACELBRA, 2004).

Esta revisão teve como objetivo levantar informações sobre a produção de pães sem glúten, com ênfase nos aspectos tecnológicos e nutricionais que possibilitam o desenvolvimento de produtos com melhores propriedades, podendo contribuir para a maior adesão dos pacientes celíacos ao tratamento.

\section{ASPECTOS TECNOLÓgICOS}

\subsection{MATÉRIAS-PRIMAS}

As proteínas encontradas nos grãos podem ser classificadas de acordo com a função exercida, ou seja, estrutural, atividade metabólica e de estocagem. As proteínas de estocagem, encontradas em abundância, são responsáveis pelas propriedades nutricionais e tecnológicas dos grãos. São classificadas de acordo com a sua solubilidade em solventes, nas seguintes frações proteicas: albuminas (solúveis em água), globulinas (solúveis em solução salina), prolaminas (solúveis em solução alcoólica) e glutelinas (solúveis em solução alcalina ou ácida fraca). As proporções dessas frações variam muito entre os cereais, conferindo diferentes características físicas, químicas, funcionais e nutricionais aos grãos (MARCONE, KAKUDA e YADA, 1998a, 1998b).

A fração proteica, encontrada em maior quantidade entre as plantas pertencentes à classe das monocotiledôneas (trigo, centeio, sorgo, cevada e milho), são as prolaminas seguidas pelas gluteninas. Somente na aveia e no arroz as gluteninas apresentam-se em maior quantidade, seguidas pelas globulinas. Já nas dicotiledôneas, a principal fração é a globulina (MARCONE, KAKUDA e YADA, 1998b, 1998c).

Pesquisas envolvendo a caracterização e a quantificação das frações proteicas dos grãos são realizadas com vários objetivos, dentre os quais o de determinar o conteúdo de glúten. Por um lado, a presença de glúten traz importância tecnológica para o grão, mas por outro, sua ausência constitui aspecto positivo muito relevante para os portadores da doença celíaca. A Comissão do 
Codex Alimentarius (Working Group on Prolamin Analysis and Toxicity) definiu glúten como: "aquelas proteínas, comumente encontradas no trigo, tricale, centeio, cevada ou aveia, às quais algumas pessoas são intolerantes". Estabeleceram, também, o limite máximo de $20 \mathrm{ppm}$ para que o alimento seja considerado isento de glúten (CODEX ALIMENTARIUS, 2003).

A farinha de arroz constitui o substituto da farinha de trigo mais utilizado em razão de apresentar sabor suave e cor branca. Outros ingredientes empregados, frequentemente, são farinhas e amidos à base de outros cereais e tubérculos, como o milho, a batata e a mandioca.

\subsection{NOVOS INGREDIENTES}

O glúten, responsável pelas propriedades de extensibilidade, elasticidade, viscosidade e retenção de gás da massa contribui para a aparência e estrutura do miolo dos pães. Por isso, a obtenção de produtos isentos de glúten torna-se tecnologicamente difícil, sendo muitas vezes necessária a combinação de diversos ingredientes e alteração dos processos tradicionais. A massa sem glúten não tem capacidade de reter o gás gerado durante a fermentação e o forneamento, originando pão com baixo volume específico e miolo firme e borrachento (RANHOTRA, LOEWE e PUYAT, 1975; NISHITA, ROBERTS e BEAN, 1976; YLIMAKI et al., 1991; MOORE et al., 2004).

Para a substituição da farinha de trigo tem sido utilizada principalmente a farinha de arroz, que também pode ser combinada com farinhas e amidos à base de outros cereais e tubérculos. Devido a diferente proporção das frações de proteínas de estocagem, a farinha de arroz é incapaz de desenvolver rede proteica similar ao glúten. Por isso, aditivos como hidrocoloides, emulsificantes, produtos lácteos, proteínas, amido gelatinizado e enzimas têm sido utilizados visando melhorar as qualidades reológicas da massa, o volume final, as características estruturais e de textura, bem como a vida-de-prateleira de pães sem glúten (GALLAGHER, GORMLEY e ARENDT, 2004).

Os hidrocoloides aumentam a viscosidade da massa, melhorando a capacidade de retenção de gás e gerando produtos com maior volume e miolos com melhores características estruturais e de textura (YLIMAKI et al., 1991; GALLAGHER, GORMLEY e ARENDT, 2004). Vários hidrocoloides como hidroxipropilmetil-celulose, carboximetil-celulose, gomas locuste, guar e xantana, pectina, $\beta$-glucana, estão sendo investigadas visando a melhoria das propriedades dos pães sem glúten (YLIMAKI et al., 1991; SÁNCHEZ, OSELLA e DE LA TORRE, 2002; GALLAGHER, GORMLEY e ARENDT, 2003; McCARTHY et al., 2005; SCHOBER et al., 2005; GAMBUS, SIKORA e ZIOBRO, 2007; LAZARIDOU et al., 2007; DEMIRKESEN et al., 2010). No geral, os hidrocoloides são adicionados em níveis que variaram de 1 a $4 \%$ (base farinha) e os melhores resultados em termos de volume e textura foram obtidos pela incorporação de 1 a $2 \%$. Os resultados são variáveis em função da formulação e das condições de processamento.

Ingredientes proteicos como a farinha de soja, o isolado proteico de soja, o leite, e o isolado proteico do leite foram utilizados para melhorar a aparência, o sabor e as características do miolo de pães sem glúten, podendo contribuir também para aumentar o valor nutricional das formulações (RANHOTRA, LOEWE e PUYAT, 1975; SÁNCHEZ, OSELLA e DE LA TORRE, 2002, 2004; GALLAGHER et al., 2003; RIBOTTA et al., 2004). Os efeitos da incorporação de ingredientes proteicos podem ser melhorados pela adição de transglutaminase, enzima que catalisa reações entre proteínas, podendo formar redes proteicas, o que contribui para a estrutura de pães sem glúten. Moore et al. (2006) e Renzetti, Bello e Arendt (2008) observaram que o efeito da transglutaminase nas propriedades do pão sem glúten depende dos níveis de enzima adicionados e também da fonte proteica presente na formulação.

Os efeitos combinados da incorporação de hidrocoloides, proteínas e enzimas também resultaram na melhoria das propriedades do pão sem glúten. Marco e Rosell (2008) observaram aumento de volume e redução da dureza do miolo de formulações à base de farinha de arroz, após a inclusão de isolado proteico de soja, hidroxipropilmetil-celulose e transglutaminase. Gujral e Rosell (2004) observaram que a adição de glicose oxidase possibilita a redução das concentrações de hidroxipropilmetilcelulose, gerando pão com maior volume específico. Outra abordagem que vem sendo investigada é a adição de bactérias lácteas e a extensão do tempo de fermentação. Os resultados mostraram melhoria da textura e do sabor do pão sem glúten durante sua vida-deprateleira (MOORE et al., 2007; MOORE, BELLO e ARENDT, 2008). 
A gelatinização do amido constitui um dos pré-requisitos para estabelecer a rede tridimensional capaz de reter gases e de se expandir durante a fermentação e o forneamento do pão sem glúten. Alguns autores observaram que a incorporação de farinhas gelatinizadas obtidas por extrusão, 10\% de farinha de arroz (CLERICI e EL-DASH, 2006) e 50\% de farinha de milho e soja (CURIC et al., 2007), ocasionou aumento do volume e melhorou a aparência das formulações.

Pães isentos de glúten apresentam reduzida vida-de-prateleira em relação aos pães com trigo, pois a estrutura elástica formada pelas proteínas desnaturadas do glúten ao redor do amido minimiza os efeitos da retrogradação do amido e retarda o ressecamento do miolo (GALLAGHER et al., 2003; MOORE et al., 2004). Para a panificação convencional estão disponíveis vários condicionadores de processo e melhoradores que contribuem para as características do produto final e prolongam sua vida-de-prateleira. Muitos desses ingredientes são baseados na combinação de enzimas, emulsificantes, hidrocoloides e outros aditivos (SLUIMER, 2005). A farinha de arroz apresenta menor resposta aos condicionadores de massa e enzimas que a farinha de trigo, possivelmente devido à natureza hidrofóbica das proteínas (NISHITA, ROBERTS e BEAN, 1976; GUJRAL et al., 2003). Gujral et al. (2003) e Gujral, Haros e Rosell (2003) observaram que a adição de ciclodextranase na formulação do pão de arroz ocasionou aumento do volume específico, melhora da textura do miolo e diminuição da retrogradação da amilopectina durante o armazenamento.

As pesquisas indicam que os hidrocoloides, bem como os agregados proteicos obtidos por ação da transglutaminase, podem contribuir para a maior retenção de umidade e maciez do miolo durante o armazenamento, prolongando a vida-de-prateleira de pães sem glúten (MOORE et al., 2006; ONYANGO et al., 2009). Estudos recentes vêm mostrando que a adição de emulsificantes pode auxiliar a manutenção da maciez do miolo e, consequentemente, contribuir para a extensão da vida útil de pães sem glúten (NUNES et al., 2009; ONYANGO UBENHND e LINDHAUER, 2009).

\section{ASPECTOS NUTRICIONAIS}

Produtos isentos de glúten geralmente são elaborados com farinhas ou amidos refinados e, portanto, apresentam baixos teores de fibras e de micronutrientes. Assim, a fortificação dessas formulações e a utilização de matérias-primas com valor nutricional e funcional vem sendo recomendada (KUPPER, 2005; THOMPSON et al., 2005).

Visando melhorar o valor nutricional de pães sem glúten foi realizada a fortificação com ferro (KISKINI et al., 2007), a suplementação com lisina e treonina para a complementação do seu perfil de aminoácidos (GAMBUS, GAMBUS e SABAT, 2002), a incorporação de fibras isoladas (KORUS e ACHREMOWICZ, 2004; SABANIS, LEBESI e TZIA, 2009; WITCZAK et al., 2010), amido resistente (KORUS et al., 2009) e das fibras prebióticas inulina e oligofrutose (KORUS et al., 2006; CAPRILES, 2009).

A incorporação de soja, leite (SÁNCHEZ, OSELLA e DE LA TORRE, 2002 e 2004), amaranto (GAMBUS, GAMBUS e SABAT, 2002; CAPRILES, 2009; ALVAREZ-JUBETE et al., 2010), quinoa (ALVAREZ-JUBETE et al., 2010), trigo sarraceno (PRUSKA-KEDZIOR et al., 2008; ALVAREZ-JUBETE et al., 2010), cereais integrais (MOORE et al., 2004), sorgo (SCHOBER et al., 2005) e linhaça (GAMBUS, 2005) contribuíram para o aumento do teor de macro e micro nutrientes, diversificando as características sensoriais de formulações de pães sem glúten.

\section{CONCLUSÃO}

A doença celíaca não tem cura e apresenta como único tratamento a remoção do glúten da dieta. Dessa forma, a elaboração de produtos específicos é essencial para melhoria da qualidade de vida desses pacientes. Avanços têm sido observados na tecnologia de panificação sem glúten, podendo ser obtidos produtos com características sensoriais adequadas e de elevado valor nutricional. O desafio agora é a ampliação da comercialização desses produtos e a redução de custos de forma a torná-los acessíveis para a população celíaca, contribuindo para maior adesão ao tratamento. 


\section{ABSTRACT \\ ADVANCES IN THE FORMULATION OF GLUTEN-FREE BREADS: TECHNOLOGICAL AND NUTRITIONAL ASPECTS}

The present review aimed to gather information concerning formulation of gluten-free breads, highlighting technological and nutritional aspects. Technological approaches included the use of starches, hydrocolloids, proteins, enzymes, emulsifiers and combinations to improve the structure, the texture, the acceptability and the shelf-life of gluten-free breads. Were presented researches about the addition of dietary fibers, proteins, minerals and whole grains flours in order to improve the nutritional value of these products. It was verified the need of increasing research and development in this area, in order to make gluten-free bread, with good technological and nutritional properties, more available to celiac disease sufferers, contributing to a better compliance to a strict gluten-free diet and improving the quality of life of these patients.

KEY-WORDS: CELIAC DISEASE; GLUTEN-FREE DIET; GLUTEN-FREE BREAD.

\section{REFERÊNCIAS}

1 ACELBRA. Associação de Celíacos do Brasil. Dados estatísticos. Disponível em: http://www.acelbra.org.br/2004/ estatisticas.php Acesso em: 20 out. 2008.

2 ALVAREZ-JUBETE, L.; AUTY, M.; ARENDT, E.K.; GALLAGHER, E. Baking properties and microstructure of pseudocereal flours in gluten-free bread formulations. European Food Research and Technology, v.230, n.3, p.437445,2010

3 ANDREOLI, C.S. Hábito alimentar e estado nutricional de pacientes e de familiares com doença celíaca. 2003. 68 p. Dissertação (Mestrado em Nutrição) Escola Paulista de Medicina da Universidade Federal de São Paulo, São Paulo, 2003.

4 CAPRILES, V. D. Otimização de propriedades nutricionais e sensoriais de produtos à base de amaranto enriquecidos com frutanos, para intervenção em celíacos. 2009. 199 p. Tese (Doutorado em Ciências) Faculdade de Saúde Pública da Universidade de São Paulo, São Paulo, 2009.

5 CATASSI, C.; BEARZI, I.; HOLMES, G.K.T. Association of celiac disease and intestinal lymphomas and other cancers. Gastroenterology, v.128, n.4, p.S79-S86, 2005.

6 CLERICI, M.T.P.S.; EL-DASH, A. A. Farinha extrusada de arroz como substituto de glúten na produção de pão de arroz. Archivos Latinoamericanos de Nutrición, v.56, n.3, p.288-298, 2006.

7 CODEX ALIMENTARIUS COMISSION. Report of the twenty-five session of the Codex Committee on Nutrition and Foods for Special Dietary Uses - Alinorm 04/27/26. Appendix III - Draft revised standard for gluten free-foods. Bonn, Germany, 3-7 November 2003. Available at: http://www.fao.org/docrep/meeting/008/j1464e/j1464e01.htm\#P49_12812 Access at: 20 out. 2008 .

8 CURIC, D.; NOVOTNI, D.; TUSAK, D.; BAUMAN, I.; GABRIC, D. Gluten-free bread production by the corn meal and soybean flour extruded blend usage. Agriculturae Conspectus Scientificus, v.72, n.3, p.227-232, 2007.

9 D'AMICO, M.A.; HOLMES, J.; STAVROPOULOS, S.N.; FREDERICK, M.; LEVY, J.; DEFELICE, A.R.; KAZLOW, P.G.; GREEN, P.H.R. Presentation of pediatric celiac disease in the United States: prominent effect of breastfeeding. Clinical Pediatrics, v.44, n.3, p.249-258, 2005.

10 DEMIRKESEN, I.; MERT, B.; SUMNU, G.; SAHIN, S. Rheological properties of gluten-free bread formulations. Journal of Food Engineering, v.96, n.2, p.295-303, 2010.

11 EGASHIARA, E.M.; ALMEIDA, O.F.; BARBIERI, D.; KODA, Y.K.L.O. O celíaco e a dieta: problemas de adaptação e alimentos alternativos. Pediatria, v.8, p.41-44, 1996.

12 FERGUSON, A.; KINGSTONE, K. Celiac disease and malignancies. Acta paediatrica supplementum, v.85, n.412, p.7881, 1996.

13 GALLAGHER, E.; GORMLEY, T.R.; ARENDT, E.K. Crust and crumb characteristics of gluten free breads. Journal of Food Engineering, v.56, n.2, p.153-161, 2003.

14 GALLAGHER, E.; KUNKEL, A.; GORMLEY, T.R.; ARENDT, E.K. The effect of dairy and rice powder addition on loaf and crumb characteristics, and on shelf life (intermediate and long-term) of gluten-free breads stored in a modified atmosphere. European Food Research and Technology, v.218, n.1, p.44-48, 2003. 
15 GALLAGHER, E.; GORMLEY, T.R.; ARENDT, E.K. Recent advances in the formulation of gluten-free cereal based products. Trends in Food Science \& Technology, v.15, n.3, p.143-152, 2004.

16 GAMBUS, H.; GAMBUS, F.; SABAT, R. The research on quality improvement of gluten-free bread by amaranthus flour addition. Zywnosc, v.9, p.99-112, 2002.

17 GAMBUS, H. Linseed (Linun usitatissimimun L) as a source of nutrients in gluten-free bread. Zywnosc, v.45, p.61-74, 2005.

18 GAMBUS, H.; SIKORA, M.; ZIOBRO, R. The effect of composition of hydrocolloids on properties of gluten-free bread. ACTA Scientiarum Polonorum - Technologia Alimentaria, v.6, n.3, p.61-74, 2007.

19 GUJRAL, H.S.; GUARDIOLA, I.; CARBONELL, J.V.; ROSELL, C.M. Effect of cyclodextrinase on dough rheology and bread quality from rice flour. Journal of Agricultural and Food Chemistry, v.51, n. 13, p.3814-3818, 2003.

20 GUJRAL, H.S.; HAROS, M.; ROSELL, C.M. Starch hydrolyzing enzymes for retarding the staling of rice brad. Cereal Chemistry, v.80, n.6, p.750-754, 2003.

21 GUJRAL, H.S.; ROSELL, C.M. Improvement of the breadmaking quality of rice flour by glucose oxidase. Food Research International, v.37, n.1, p.75-81, 2004.

22 HOFFENBERG, E.J.; MACKENZIE, T.; BARRIGA, K.J.; EISENBARTH, G.S.; BAO, F.; HAAS, J.E.; ERLICH, H.; BUGAWAN, T.L.T.; SOKOL, R.J.; TAKI, I.; NORRIS, J.M.; REWERS, M. A prospective study of the incidence of childhood celiac disease. Journal of Pediatrics, v.143, n.3, p. 308-314, 2003

23 KISKINI, A.; ARGIRI, K.; KALOGEROPOULOS, M.; KOMAITIS, M.; KOSTAROPOULOS, A.; MANDALA, I.; KAPSOKEFALOU, M. Sensory characteristics and iron dialyzability of gluten-free bread fortified with iron. Food Chemistry, v.102, n.1, p.309-316, 2007.

24 KORUS, J.; ACHREMOWICZ, B. Fiber preparations of different origin used as additives in baking gluten-free breads. Zywnosc, v.38, p.65-73, 2004.

25 KORUS, J.; GRZELAK, K.; ACHREMOWICZ, K.; SABAT, R. Influence of prebiotic additions on the quality of gluten-free bread and on the content of inulin and fructooligosaccharides. Food Science and Technology International, v.12, n.6, p.489-495, 2006.

26 KORUS, J.; WITCZAK, M.; ZIOBRO, R.; JUSZCZAK, L. The impact of resistant starch on characteristics of gluten-free dough and bread. Food Hydrocolloids, v.23, n.3, p.988-995, 2009.

27 KUPPER, C. Dietary guidelines and implementation for celiac disease. Gastroenterology, v.128, n.4, p.S121-S127, 2005.

28 LAZARIDOU, A.; DUTA, D.; PAPAGEORGIOU, M.; BELC, N.; BILIADERIS, C.G. Effects of hydrocolloids on dough rheology and bread quality parameters in gluten-free formulations. Journal of Food Engineering, v.79, n.3, p.1033-1047, 2007.

29 LEE, A.; NEWMAN, J. Celiac diet: its impact on quality of life. Journal of the American Dietetic Association, v.103, n.11, p.1533-1535, 2003.

30 MARCO, C.; ROSELL, C.M. Breadmaking performance of protein enriched, gluten-free breads. European Food Research and Technology, v.227, n.4, p.1438-1447, 2008.

31 MARCONE, M.F.; KAKUDA, Y.; YADA, R.Y. Salt-soluble seed globulins of dicotyledonous and monocotyledonous plants I. Isolation/ purification and characterization. Food Chemistry, v.63, n.1, p.27-47, 1998a.

32 MARCONE, M.F.; KAKUDA, Y.; YADA, R.Y. Salt-soluble seed globulins of dicotyledonous and monocotyledonous plants II. Isolation/ purification and characterization. Food Chemistry, v.63, n.2, p.265-274, 1998b.

33 MARCONE, M.F.; KAKUDA, Y.; YADA, R.Y. Immonochemical examination of the surface physico-chemical properties of various dicotyledonous and monocotyledonous globulin seed storage protein. Food Chemistry, v.63, n.1, p.85-95, 1998c.

34 McCARTHY, D.F.; GALLAGHER, E.; GORMLEY, T.R.; SCHOBER, T.J.; ARENDT, E.K. Application of response surface methodology in the development of gluten-free bread. Cereal Chemistry, v.82, n.5, p.609-615, 2005.

35 MOORE, M.M.; SCHOBER, T.J.; DOCKERY, P.; ARENDT, E.K. Textural comparisions of gluten-free and wheat-based doughs, batters, and breads. Cereal Chemistry, v.81, n.5, p.567-575, 2004.

36 MOORE, M.M.; HEINBOCKEL, M.; DOCKERY, P.; ULMER, H.M.; ARENDT, E.K. Network formation in gluten-free bread with application of transglutaminase. Cereal Chemistry, v.83, n.1, p.28-36, 2006. 
37 MOORE, M.M.; JUGA, B.; SCHOBER, T.J.; ARENDT, E.K. Effect of lactic acid bacteria on properties of gluten-free sourdoughs, batters, and quality and ultrastructure of gluten-free bread. Cereal Chemistry, v.84, n.4, p.357-364, 2007.

38 MOORE, M.M.; BELLO, F.D.; ARENDT, E.K. Sourdough fermented by Lactobacillus plantarum FST 1.7 improves the quality and shelf life of gluten-free bread. European Food Research and Technology, v.226, n.6, p.1309-1316, 2008.

39 NISHITA, K.D.; ROBERTS, R.L.; BEAN, M.M. Development of a yeast-leavened rice-bread formula. Cereal Chemistry, v.53, n.5, p.626-635, 1976.

40 NUNES, M.H.B.; MOORE, M.M.; RYAN, L.A.M.; ARENDT, E.K. Impact of emulsifiers on the quality and rheological properties of gluten-free breads and batters. European Food Research and Technology, v.228, n.4, p.633-642, 2009.

41 ONYANGO, C.; MUTUNGI, C.; UNBEHEND, G.; LINDHAUER, M. G. Creep-recovery parameters of gluten-free batter and crumb properties of bread prepared from pregelatinised cassava starch, sorghum and selected proteins. International Journal of Food Science and Technology, v.44, n.12, p.2493-2499, 2009.

42 ONYANGO, C.; UNBEHEND, G.; LINDHAUER, M. G. Effect of cellulose-derivatives and emulsifiers on creep-recovery and crumb properties of gluten-free bread prepared from sorghum and gelatinised cassava starch. Food Research International, v.42, n.8, p.949-955, 2009.

43 PRUSKA-KEDZIOR, A.; KEDZIOR, Z.; GORACY, M.; PIETROWSKA, K.; PRZYBYLSKA, A.; SPYCHALSKA, K. Comparison of rheological, fermentative and baking properties of gluten-free dough formulations. European Food Research and Technology, v.227, n.5, p.1523-1536, 2008

44 RANHOTRA, G.S.; LOEWE, R.J.; PUYAT, L.V. Preparation and evaluation of soy-fortified gluten-free bread. Journal of Food Science, v.40, n.1, p.62-64, 1975

45 RASHID, M.; CRANNEY, A.; GRAHAM, I.; ZARKADAS, M.; SWITZER, C.; CASE, S.; MALLOY, M.; WARREN, R.; BUTZNER, D. Canadian celiac health survey: pediatric data. Journal of Pediatric Gastroenterology \& Nutrition, v.37, n.3, p.369-371, 2003.

46 RENZETTI, S.; BELLO, F.D.; ARENDT, E.K. Microstructure, fundamental rheology and baking characteristics of batters and breads from different gluten-free flours treated with a microbial transglutaminase. Journal of Cereal Science, v.48, n.1, p.33-45, 2008

47 REWERS, M. Epidemiology of celiac disease: what are the prevalence, incidence, and progression of celiac disease? Gastroenterology, v.128, n.4, p.S47-S51, 2005.

48 RIBOTTA, P.D.; AUSAR, S.F.; MORCILLO, M.H.; PÉREZ, G.T.; BELTRAMO, D.M.; LEON, A.E. Production of gluten-free bread using soybean flour. Journal of the Science of Food and Agriculture, v.84, n.14, p.1969-1974, 2004.

49 SABANIS, D.; LEBESI, D.; TZIA, C. Effect of Dietary fiber enrichment on selected properties of gluten-free bread. LwtFood Science and Technology, v.42, n.8, p.1380-1389, 2009.

50 SÁNCHEZ, H.D.; OSELLA, C.A.; DE LA TORRE, M. A. Optimization of gluten-free bread prepared from cornstarch, rice flour and cassava starch. Journal of Food Science, v.67, n.1, p.416-419, 2002

51 SÁNCHEZ, H.D.; OSELLA, C.A.; DE LA TORRE, M. A. Use of response surface methodology to optimize gluten-free bread fortified with soy flour and dry milk. Food Science and Technology International, v.10, n.1, p.5-9, 2004.

52 SÁNCHEZ, M.C.; CARRASCO, A.V.; GARCIA, J.I.; AGUIRRE, A.S.; COMENZANA, J.C.V.; TOBIO, P.M.; STIENLET, L.L. Enfermedad celíaca y talla baja en niños. Anales Españoles de Pediatria, v.37, n.4, p.304-306, 1992.

53 SCHOBER, T.J.; MESSERSCHMIDT, M.; BEAN, S.R.; PARK, S.H.; ARENDT, E.K. Gluten-free bread from sorghum: quality differences among hybrids. Cereal Chemistry, v.82, n.4, p.394-404, 2005.

54 SLUIMER, P. Principles of breadmaking: functionality of raw materials and process steps. Saint. Paul: American Association of Cereal Chemists, 2005. p.49-79.

55 THOMPSON, T.; DENNIS, M.; HIGGINS, L.A.; LEE, A.R.; SHARRETT, M.K. Gluten-free diet survey: are Americans with celiac disease consuming recommended amounts of fiber, iron, calcium and grain foods? Journal of Human Nutrition and Dietetics, v.18, p.163-169, 2005.

56 YLIMAKI, G.; HAWRYSH, Z.J.; HARDIN, R.T.; THOMSON, A.B.R. Response surface methodology to the development of rice flour yeast breads: sensory measurements. Journal of Food Science, v.56, n.3, p.751-757, 1991.

57 WITCZAK, M.; KORUS, J.; ZIOBRO, R.; JUSZCZAK, L. The effects of maltodextrins on gluten-free dough and quality of bread. Journal of Food Engineering, v.96, n.2, p. 258-265, 2010. 


\section{AGRADECIMENTOS}

À Fundação de Amparo a Pesquisa do Estado de São Paulo pelas bolsas de estudo 2004/14127-3 e 2010/05144-2. 